\title{
A UTILIZAÇÃO DAS FIBRAS DE REFORÇO NA ODONTOLOGIA
}

\section{THE USE OF FIBER-REINFORCED COMPOSITES IN DENTISTRY}

\author{
Priscila Paiva Portero $^{1 *}$, Patricia Grau Grullón ${ }^{1}$, Rafael Gomes Ditterich ${ }^{1}$, \\ Osnara Maria Mongruel Gomes ${ }^{1}$, João Carlos Gomes ${ }^{1}$ \\ 1* Autor para contato: Universidade Estadual de Ponta Grossa - UEPG, Programa de \\ Mestrado em Odontologia, Campus em Uvaranas, Ponta Grossa, PR, Brasil; \\ (18) 36415881; e-mail: priscis.portero@ig.com.br
}

Recebido para publicação em 16/11/2005

Aceito para publicação em 07/02/2006

\section{RESUMO}

Este artigo revisa a literatura sobre as fibras de reforço utilizadas em odontologia, os tipos de fibras, a arquitetura e as indicações para seu uso, visto que são materiais relativamente recentes, sendo importante que os profissionais e estudantes conheçam suas indicações, benefícios e limitações.

Palavras-chave: compostos, materiais dentários, resistência

\begin{abstract}
This article reviews studies on fiber-reinforced composites used in dentistry, the types of fibers, their architecture and their indications of use, because these materials are news and it is important for dentists and students to known their indications, advantages.
\end{abstract}

Key words: composites resin, dental materials, strength

\section{Introdução}

As próteses metalocerâmicas têm sido as restaurações mais populares em próteses fixas durante os últimos 40 anos. O sucesso delas está baseado em sua longevidade clínica e estética aceitável, embora nas margens esta fique prejudicada pela coloração acin- zentada ou escurecida devido à presença do metal. Entretanto, têm sido discutidos também os efeitos alérgicos ou tóxicos das ligas metálicas, incentivando os pesquisadores a desenvolver próteses sem metal.

As fibras de reforço foram introduzidas na odontologia devido a propriedades como por exemplo a boa resistência flexural e outras características físicas 
desejáveis de um material de infra-estrutura de prótese fixa (Freilich et al., 2000). Além disso, a infra-estrutura dos compósitos reforçados por fibras (FRCs) é translúcida e não precisa de material opaco, o que permite que haja uma camada mínima de resina composta particulada de recobrimento e uma excelente estética. A infra-estrutura de FRC fotopolimerizada possui uma camada inibida pelo oxigênio, pegajosa em sua superfície externa que permite uma ligação química direta com o compósito de recobrimento, eliminando a necessidade de retenção mecânica que seria necessária com uma estrutura metálica (Freilich et al., 2001).

Este é um trabalho de revisão de literatura que possui o propósito de apresentar os fundamentos básicos, propriedades, composições e aplicações clínicas das variadas fibras de reforço presentes no mercado odontológico.

\section{Revisão de Literatura}

As fibras de reforço têm sido discutidas na literatura odontológica desde 1960, embora apenas recentemente estes produtos têm tido o reconhecimento para seu uso clínico. Apresentam procedimentos atrativos para restauração de espaços protéticos associadas à materiais resinosos, pois apresentam boas propriedades físicas, estéticas e durabilidade (Cho et al., 2002).

O efeito da concentração de fibras na resistência à fratura da base de resina acrílica foi estudado por Vallittu (1994). Como conclusão foi apresentado que as fibras incorporadas a resina acrílica aumentaram a resistência à fratura dos corpos-de-prova. Com a concentração mais alta de fibras houve aumento da resistência e o uso de fibras como reforço causou a formação de espaços vazios dentro do sistema matriz da fibra, desconhecendo-se o que ocasionou a formação desses espaços.

Dando continuidade ao trabalho anterior Vallittu (1994) realizou um estudo para determinar o efeito da contração de polimerização do poli-metacrilado (resina) aplicado ao fio de fibra em força transversal. Concluiu que: 1) quanto menor for a contração de polimerização do PMMA (polimetil-metacrilato), dentro do fio da fibra, há um aumento de resistência à fratura do composto fibra-resina; 2) a contração de polimerização pode ser diminuída, imergindo-se as fibras na mistura PMMA fornecendo uma camada mais grossa de resina acrílica na superfície da fibra de vidro, quando comparada com a camada fornecida pelo monômero líquido.

A resistência de uma prótese parcial removível de resina acrilica reforçada com fibra de vidro contínua e outra reforçada com fio metálico foi avaliada por Vallittu (1996), os resultados mostraram que as fraturas nos espécimes reforçados com metal ocorrem coincidentemente na interface da resina com o fio, o mesmo não ocorre com a fibra de vidro e sim com o lado oposto da amostra, concluindo que a resistência do reforço das próteses com fibra de vidro são maiores que o reforço das próteses com fio metálico convencional.

Uma resina com fibras de vidro contínuas sob a ação de forças de impacto que poderiam ser acidentais ou forças mastigatórias foi testada por Vallittu e Katja (1997). Neste estudo mostraram que a absorção de energia de impacto através da resiliência das fibras, aumenta a energia elástica da resina, resultando no aumento considerável do reforço da resina.

Samadzadeh et al. (1997) avaliou o efeito das fibras trançadas de polietileno (Ribbond) com e sem dois tipos de reforço na resistência à fratura. O primeiro reforço foi o PMMA (polimetil-metacrilato) e o segundo Provipont DC (uma resina de dupla presa). Concluíram que as fibras de polietileno tratadas e reforçadas com PMMA não mostraram aumento significante na força de fratura quando comparadas com as fibras sem reforço. O reforço de resina de dupla presa revelaram resistência à fratura significativamente mais alta quando comparadas com as fibras sem reforço de resina e de PMMA nas restaurações.

A força de fratura de prótese parcial fixa de três elementos posterior reforçados por fibras foi estudada por Loose et al. (1998). Os materiais analisados foram o In-Ceram e o sistema Targis/Vectris com e sem retentor confeccionados pelo fabricante. Os resultados mostraram que o sistema Targis/Vectris mostrou uma resistência à fratura significativamente maior que o sistema In-Ceram.

Segundo Felippe et al. (2001) o propósito básico do uso de fibras em odontologia é reforçar um 
grande volume ou uma grande extensão de resina acrílica ou qualquer outro material com características químicas e problemas clínicos semelhantes aos das resinas. Outra vantagem de estruturas de resina reforçadas por fibra é a possibilidade de reparos existentes pela fratura da resina aplicada sobre a fibra (Kallio; Lastumáki e Vallittu, 2000).

As fibras de reforço, de acordo com Felippe $e t$ al. (2001), se apresentam sob a forma de fitas ou cordões maleáveis, possuem como vantagens: alta resistência quando agrupados, os fios apresentam translucidez e em certos casos transparência, grande maleabilidade dos produtos facilitando sua aplicação clínica, leveza e ausência de oxidação quando comparadas com as estruturas metálicas.

As fibras de reforço atuam nas resinas compostas de maneira semelhante à armação metálica no concreto. Uma ampla estrutura de resina também precisa de reforço no seu interior, que pode ser feito com metal ou com materiais mais atuais como as fibras (Vallittu e Katja, 1997). Em alguns casos as fibras podem ser incorporadas às resinas para melhorar sua consistência e facilitar assim o seu uso. As fibras distribuem e dissipam a força na estrutura à qual foi incorporada, diminuindo e homogeneizando a tensão transmitida aos apoios, também pode servir como transmissores ópticos dentro de uma estrutura, conduzindo luz (Felippe et al., 2001) o que as torna muito vantajosas na confecção de núcleos intra-radiculares.

Cho et al. (2002) avaliaram a resistência à fratura de cerômeros reforçados por fibras. Os autores realizaram três preparos oclusais com ângulos convergentes (6, 10 e 15 graus). Foram feitos 30 corpos de provas divididos em 3 grupos restaurados com Targis/Vectris. Os corpos de provas foram levados a máquina universal de testes e submetidos à teste de compressão. Os resultados foram analisados com teste estatístico de Kruskall-Wallis com um índice de variância de 0,05. A angulação de 6 graus teve alta resistência à fratura (1543N). A resistência à fratura das demais coroas foram em torno de 1390N. O mapeamento do microscópio eletrônico mostrou que as coroas exibiram duas fases de fraturas : rachar-se e fragmentar-se na camada entre o adesivo e o Vectris. Concluíram que dentro das limitações do experimento, que diminuindo a convergência do ângulo axial das coroas de cerômeros reforçados por fibras aumenta a resistência à fratura.

Meiers; Kazemi e Donádio (2003) avaliaram o efeito dos compósitos reforçados por fibras pré-impregnadas e não pré-impregnadas na força de cizalhamento do esmalte condicionado. Para isso, utilizou 12 espécimes de dentes bovinos divididos em cinco grupos, G1 controle (compósito sem reforço de fibras). G2= Ribbond, G3= Splint-It Unidirecional, G4= Splint-It Woven e G5= Connect. As fibras foram aderidas aos espécimes com sistema adesivo Prime \&Bond e uma camada resina composta Tetric flow . Os espécimes foram termociclados 1000 vezes entre $5^{\circ}$ e $55^{\circ} \mathrm{C}$ e levados a uma máquina de testes universal em força de cizalhamento, aumentando a carga linearmente até ocorrer a fratura (MPa). As superfícies fraturadas não aderidas foram avaliadas para determinar a natureza da fratura Os resultados em MPa foram os seguintes: $\mathrm{G} 1=15,6 \pm 2,4 ; \mathrm{G} 2=15,8 \pm 2,2 ; \mathrm{G} 3=15,3 \pm 2,4$; $\mathrm{G} 4=16,5 \pm 1,8 ; \mathrm{G} 5=18.8 \pm 1,5$. O G5 teve um melhor desempenho em relação aos demais. A análise de variança revelou que as falhas se deram por fraturas coesivas nas fibras Splint-It Unidirecional e Connect. Fraturas adesivas ocorreram nas fibras na interface da fibra de reforço Splint-It Woven. Conclúram com esse estudo que não há nenhuma diferença na força de cizalhamento adesivo das fibras testadas com o grupo controle com exceção da fibra Connect que ofereceu valores de resistência relativamente mais altos.

Lassila e Vallittu (2004) investigaram a influência de uma quantidade relativamente baixa de fibras nas propriedades flexurais de uma estrutura de compósito reforçado por fibras (FRC) e determinaram o conteúdo de monômero residual dos corpos-de-prova após diferentes condições de polimerização por luz. Os autores concluíram que: 1) o conteúdo de monômero foi mais baixo nos corpos-de-prova polimerizados com aparelho com mais alta temperatura de polimerização e intensidade de luz; 2) para se otimizar a resistência flexural e uma construção de FRC com baixa fração de fibras, as fibras deveriam ser posicionadas no lado de tração dos corpos-de-prova.

Carvalho et al. (2005) avaliaram a resistência à flexão de duas resinas compostas, uma microhíbrida (Filtek ${ }^{\mathrm{TM}}$ Z-250-3M/ESPE) e outra de micropartícula (Durafill VS - Heraues Kulzer) com diferentes fontes de ativação, comparando os dispositivos de LED 
(Ultrablue-DMC e L.E.Demetron - Kerr) e um fotoativador de luz halógena (Optilux 501 - Kerr). De acordo com os resultados foi constatado que: 1 ) os aparelhos fotoativadores utilizados causaram comportamentos distintos das diferentes resinas compostas utilizadas; 2) as intensidades luminosas dos diferentes equipamentos foram suficientes para promover a fotoiniciação adequada das resinas compostas testadas, apesar das diferenças estatísticas encontradas; 3) a resina microhíbrida Filtek ${ }^{\mathrm{TM}} \mathrm{Z}-250$ possui resistência à flexão significativamente mais alta que a resina micropartícula Durafill VS, independente do fotoativador utilizado; 4) o aparelho LED (Ultrablue - DMC) foi mais eficiente que o L.E.Demetron para a resina composta híbrida, mas foi menos eficiente para resina de micropartícula.

\section{Tipos de Fibras}

Existem vários tipos de fibras de reforço, como por exemplo: vidro, carbono, aramida, boro, carbeto de silício, óxido de alumínio, sendo as fibras de vidro, carbono e aramida as mais utilizadas.

\section{Impregnação das Fibras}

Impregnação é a maneira com que a fibra é incorporada na matriz resinosa, sendo um dos principais fatores que influenciam na resistência. Quanto mais próxima for a união molecular entre os fios e a matriz resinosa, maior a resistência. Produtos pré-impregnados são aqueles cujo cálculo aproximado do volume de fibras na estrutura a ser reforçada já foi feita pelo fabricante, que oferece a fibra já incorporada na matriz resinosa ou epóxica. Quanto à impregnação as fibras podem ser: pré-impregnadas por resina e não préimpregnadas.

\section{Fibras de Vidro}

As fibras de vidro são constituídas de: sílica, alumínio e óxido de magnésio. São compósitos que consistem em fibras de vidro, contínuas ou descontínuas, contidas no interior de uma matriz polimérica; esse tipo de compósito é produzido nas maiores quantidades. Acomposição do vidro que é mais comu- mente estirado na forma de fibras (algumas vezes chamados de vidro E ou E-glass); Os diâmetros das fibras normalmente estão entre 3 e $20 \mathrm{~mm}$. O vidro é popular como material de reforço por ser: a) facilmente estirado na forma de fibras de alta resistência à partir do seu estado fundido; b) um material amplamente disponível e pode ser fabricado economicamente para formar um plástico reforçado com vidro, empregando-se uma ampla variedade de técnicas de fabricação de materiais compósitos; c) como uma fibra, é relativamente forte, coloca-se no interior de uma matriz de plástico produzindo um compósito que possui resistência específica muito alta; d) quando associado com diferentes plásticos, possui uma inércia química que torna o compósito útil para aplicação em meio a uma variedade de ambientes corrosivos.

As fibras de vidro são em forma de trama solta como uma trança de cabelo, apresentando espaço entre elas. (Bottino et al., 2000; Andrade et al., 2002; Kallister Junior, 2002). Por possuírem uma trama solta, quando cortadas, alteram o tamanho e desmancham, podendo determinar um reforço inconsistente quando são utilizadas.

Por essas fibras não serem impregnadas pelo fabricante, é necessário que o cirurgião-dentista ou protético realize esse procedimento antes de utilizar esses materiais como reforço. Para tanto, a fita deve ser embebida com o adesivo dentinário de metacrilato saturado de monômero.

\section{Fibras de Carbono}

O carbono é um material de fibra de alto desempenho e o reforço mais comumente utilizado em compósitos avançados com matriz polimérica (isto é, que não contém fibras de vidro). As fibras de carbono têm como vantagem a grande resistência, embora sua cor acizentada na maioria das vezes prejudique o resultado do trabalho como no caso dos núcleos de preenchimento feitos a partir de pinos de fibra de carbono, que fornecem estruturas acinzentadas. (Bottino et al., 2000; Andrade et al., 2002).

\section{Fibras de Aramida}

As fibras de aramida possuem como caracte- 
rísticas: alta resistência à fratura, alto módulo de tração, é o mais resistente de todos esses materiais sendo relativamente fracos quando submetidos à compressão, são quimicamente susceptíveis à degradação por ácidos e bases fortes, alta tenacidade, alta resistência ao impacto, alta resistência à fluência e falha por fadiga, sua coloração prejudica a estética, possui dificuldade de adaptação aos dentes. Essas fibras devem ser cortadas com tesoura especial (cerâmica). A impregnação com a resina é demorada e difícil, principalmente se a resina apresenta alta viscosidade e a fibra esgarça durante esse procedimento. (Bottino et al., 2000).

\section{Fibras de Polietileno}

As fibras de polietileno possuem coloração esbranquiçada com propriedade camaleônica, são biocompatíveis, possuem alta resistência, alto peso molecular, altamente orientada, são tratadas com plasma de gás frio, são dez vezes mais forte que o aço, podem ser trançadas, unidirecionais, entrelaçada. Essas fibras são tratadas com plasma de gás frio para permitir um aumento de sua reatividade e molhabilidade, permitindo interação química e física com as resinas compostas. Deve ser molhada com uma fina camada de resina adesiva antes de ser manipulada. $\mathrm{O}$ adesivo não deve ser monocomponente (primer + adesivo em frasco único) pela presença de primer e outros solventes orgânicos, que diminuem a reatividade da superfície do Ribbond tratado com plasma de gás frio.

O uso de agentes adesivos mais viscosos pode resultar em um incompleto molhamento das fibras. Enquanto não realizado o molhamento, essas fibras devem ser manuseadas cuidadosamente com uma pinça de algodão para evitar contaminação da superfície tratada da fibra pelo látex ou pela oleosidade da mão.

\section{Arquitetura}

A arquitetura é o dimensionamento dos fios no interior da estrutura, é importante na resistência do reforço a ser executado. A arquitetura não só define a resistência da estrutura, mas também influencia na técnica de execução.

É relevante observar que quanto mais paralela for a incidência da força em relação à disposição dos fios, maiores serão a absorção e a dissipação pela estrutura e conseqüentemente, melhor será seu desempenho (Felippe et al., 2001).

As forças atuam nos dentes em duas direções principais - axial ou paralela ao longo eixo. Isso significa que a incorporação de fibras transversais e longitudinais em odontologia é recomendada. Por possuírem alto módulo de elasticidade, essas fibras não se rompem, o que impede a fratura total da prótese quando a força aplicada sobre ela ultrapassa sua resistência à fratura da resina acrílica. Essa característica facilita o procedimento de reparo, pois não há ruptura completa da peça e a união das partes é facilmente conseguida.

A capacidade de reforço das fibras depende: Tipo de matriz (pode ser feita à partir de metais, polímeros ou cerâmica); Tipo de fibra; Distribuição das fibras; Razão fibra/matriz; Diâmetro; Comprimento, Quantidade; Orientação; Impregnação, Adesão à resina.

Para se obter o melhor desempenho mecânico das fibras deve-se levar em consideração o direcionamento das fibras, o volume de fibras na composição, a influência no comprimento da fibra (Kallister Junior, 2002).

As fibras podem ser utilizadas para substituir estruturas metálicas; apresentam melhor estética; as próteses reforçadas com fibras apresentam-se mais resistentes que as não reforçadas; podem ser utilizadas de maneira direta ou indireta; as fibras quando usadas como reforço intra-canal, minimizam a propagação de tensões na raiz, são usadas principalmente em casos de canais amplos. As próteses realizadas com reforço de fibras requerem o menor número de sessões clínicas quando comparadas com as que possuem estruturas metálicas.

\section{Indicações para o uso de Fibras de Reforço}

As principais indicações para o uso de fibras, de acordo com Felippe et al. (2001) são: núcleos de preenchimento direto, núcleos de preenchimento indireto, ferulização periodontal, ferulização ortodôntica, prótese adesiva direta e indireta, provisórios acrílicos extensos, reparo de prótese total e prótese parcial removível, coroa pura de resina composta, prótese fixa de resina composta, amplas restaurações de resina composta, resinas compostas com fibras na sua com- 
posição, diversas situações emergenciais do consultório, estruturas de próteses fixas sobre implante.

\section{Conclusões}

Com esta revisão de literatura chega-se às seguintes conclusões: a) As próteses reforçadas por fibras mostram-se mais resistentes quando comparadas às sem reforço; b) Podem ser utilizadas para substituir infra-estruturas metálicas nos casos de coroas unitárias, próteses fixas, para esplintagens e para contenções ortodônticas; c) As fibras podem ser utilizadas de maneira direta ou indireta.

\section{REFERÊNCIAS}

1. ANDRADE, M. F.; PORTO NETTO, S. T.; SAAD, J. R. C. Utilização de Fibras de reforço nas restaurações estéticas. São Paulo Artes Médicas, cap. 8, p. 151-167, 2001

2. BOTTINO, M.A. Estética em reabilitação oral Metal Free. São Paulo: Artes Médicas, cap.6, p.333-381, 2000.

3. CARVALHO, L. D.; REBELATTO, C.; CHAIN, M.; MAIA, H. P. Avaliação da resistência à flexão de resinas compostas fotoativadas por LEDs. RBO, v.62, n.1/2, p. 76-8. 2005.

4. CHO, L.; SONG, H.; KOAK, J.; HEO, Sl. Marginal accuracy and frature strength of ceromer/fiber-reinforced composite crowns: effect of variations in preparation desing. J. Prosthet. Dent., v. 88, n. 4, p. 388-95, Oct. 2002.

5. FELIPPE, L.A.; BARATIERI, L.N.; MONTEIRO JÚNIOR, S.; ANDRADA, M.A.C.; VIEIRA, L.C.C. Fibras de reforço para uso odontológico - Fundamentos e aplicações clínicas. Revista da APCD, v. 55, n. 4, jul/ago, 2001.

6. FREILICH, M. A.; KARMAKER, A. C.; BURSTONE, C. J.; GOLDBERG, A. J. Development and clinical applications of a light-polymerized fiber-reinforced composite. J. Prosthet. Dent., v. 80, n. 3, p.689-96, 1998.

7. GOMES, J. C. Odontologia estética: restaurações adesivas indiretas. São Paulo: Artes Médicas, 1996.

8. KALLIO, T. T.; LASTUMÁKI, T. M.; VALLITTU, P. K. Bonding of restorative and veneering composite resing to some polymeric composites. Dent Mater, v. 16, n.1, p. 80-6, 2000.

9. KALLISTER JUNIOR, W. D. Ciência e engenharia de materiais: uma introdução. 5. ed. São Paulo: LTC, 2002.

10. LASSILA, L. V. J.; VALLITTU, P. K. The effect of fiber position and polimerization condition on the flexural properties of fiber-reinforced composite. J. Contemp. Dent. Pract., v. 5, n. 2, p. 14-26, 2004.

11. LOOSE, M.; ROSENTRITT, M.; LEIBROCK, S.; BEHR, M.; HANDEL, G. In vitro study of fracture strength and marginal adaptation of fiber - reinforced - composite versus all ceramic fixed partial dentures. Eur. J. Prosthodont. Restor. Dent., v. 6, n. 2, p. 55-62, 1998.

12. MEIERS, J. C.; KAZEMI, R. B.; DONADIO, M. The influence of fiber reiforcement of composites on shear bond strengths to enamel. J. Prosthet. Dent., v. 89, n. 4, p.388-93, abr. 2003.

13. SAMADZADEH, A.; KUGEL, G.; HURLEY, E.; ABOUSHALA, A. Fracture strengths of provisional restorations reinforced with plasma-treated poliethylene fiber. J. Prosthet. Dent, v. 78, n. 5, p. 447-50, 1997.

14. VALLITTU, P. K. Acrylic resin - fiber composite - part Il: The effect of polymerization shrinkage of polimethyl methacrilate applied to fiber roving on tranverse strength. $\mathbf{J}$. Prosthet. Dent., v.71, n.6, p. 613-617, 1994.

15. VALLITTU, P. K. Comparison of the in vitro fatigue resistance of an acrylic resin removable partial denture reinforced with continuous glass fibers or metal wires. $\mathbf{J}$. Prosthodont., v. 5, n. 2, p. 115-21, Jun. 1996.

16. VALLITTU, P. K.; KATJA, N. Impact strength of a modified continuous glass fiber - polymethyl methacrylate. Int. J. Prost., v. 10, n. 2, p. 142-7, 1997. 